\title{
PRK convencional versus trans-PRK de un solo paso en cirugía refractiva corneal con excímer láser
}

\section{Conventional PRK versus single-step transPRK in corneal refractive surgery with excimer laser}

\author{
Giuseppe Miotto-Montesinos ${ }^{1}$, Eduardo Rojas-Alvarez ${ }^{1,2 *}$, Diego Torres-Piedra ${ }^{1}$ y Natali Torres-Palacios ${ }^{1}$

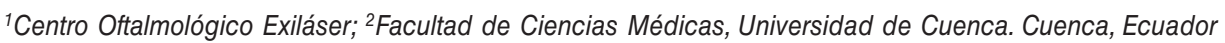

\begin{abstract}
Resumen
Objetivo: Establecer una comparación entre la técnica PRK convencional, asistida con alcohol (PRKaa), y la trans-PRK de un solo paso, en cuanto a variables clínico-refractivas y quirúrgicas. Método: Se realizó un estudio observacional, prospectivo, longitudinal, en 72 pacientes candidatos a cirugía refractiva corneal con excímer láser, con el diagnóstico de astigmatismo miópico compuesto, en el Centro Oftalmológico Exiláser, Cuenca, Ecuador, de septiembre a diciembre de 2019. Los pacientes fueron intervenidos por técnicas de superficie (trans-PRK o PRKaa). El procesamiento de los datos se realizó en el programa SPSS, versión 21.0. Para la comparación de los datos de ambas técnicas quirúrgicas se empleó la prueba de chi cuadrado, donde se consideró $p<0.05$ estadísticamente significativo. Resultados: Se obtuvo como equivalente esférico promedio para PRKaa -0.14 y para trans-PRK -0.11 , a los 3 meses del procedimiento $(p=0.34)$. El promedio de agudeza visual sin corrección obtenido fue en PRKaa de 0.93 y en trans-PRK de 0.96, sin diferencias estadísticamente significativas $(p=0.63)$. El tiempo quirúrgico para PRKaa fue de 1,080.85 s y para trans-PRK fue de $720.31 \mathrm{~s}$, menor en esta última con diferencia estadísticamente significativa $(p<0.001)$. En trans-PRK hubo menor dolor inmediatamente después de la cirugía, comparado con PRKaa $(p<0.05)$. El día promedio de cierre epitelial en PRKaa fue el 6.27 y en trans-PRK el $3.62(p=0.02)$. Conclusiones: La PRK-transepitelial de un solo paso y la PRKaa realizada en pacientes candidatos a cirugía refractiva producen resultados muy similares a los 3 meses de la cirugía en cuanto a AVSC, equivalente esférico obtenido y mínimas complicaciones postoperatorias. La trans-PRK ofrece mayores ventajas al paciente, referentes a menor tiempo quirúrgico, rápido cierre epitelial y menor dolor en el postoperatorio inmediato.
\end{abstract}

Palabras clave: PRK. Trans-PRK. Miopía. Astigmatismo. Agudeza visual. Equivalente esférico.

\section{Abstract}

Objective: To compare conventional alcohol-assisted PRK (aaPRK) and single-step transPRK in terms of clinical-refractive and surgical variables. Method: An observational, prospective, longitudinal study was carried out in 72 patients who were candidates for corneal refractive surgery with the excimer laser, with a diagnosis of compound myopic astigmatism, at the Exilaser Ophthalmological Center, Cuenca, Ecuador, from September to December 2019. Patients underwent surface surgery (transPRK or aaPRK). Data processing was performed with the SPSS program, version 21.0. For data comparison between both surgical techniques, the chi-square test was used, where $P<0.05$ was considered statistically significant. Results: The average spherical equivalent was obtained for aaPRK $(-0.14)$ and transPRK $(-0.11) 3$ months after the procedure $(p=0.34)$. UCVA average

Correspondencia:

*Eduardo Rojas-Alvarez

Federico Proaño, 4-26, y Av. Remigio Crespo Fecha de recepción: 15-02-2020

Cuenca, Ecuador

E-mail: drerojasalvarez@gmail.com

DOI: 10.24875/RMO.M20000122

0187-4519/○ 2020 Sociedad Mexicana de Oftalmología. Publicado por Permanyer. Este es un artículo open access bajo la licencia CC BY-NC-ND (http://creativecommons.org/licenses/by-nc-nd/4.0/).
Disponible en internet: 01-09-2020 Rev Mex Oftalmol. 2020;94(5):204-212 www.rmo.com.mx
Fecha de aceptación: 17-05-2020 : 
was 0.93 in aaPRK and 0.96 in transPRK, without statistically significant differences $(p=0.63)$. Surgical time was shorter in transPRK (720.31 s) compared to aaPRK (1080.85 s), with a statistically significant difference $(p<0.001)$. In transPRK there was less pain immediately after surgery, compared to aaPRK $(p<0.05)$. Epithelial closure was achieved on day 6.27 for aaPRK and in day 3.62 for transPRK (average values; $p=0.02$ ). Conclusions: Single-step transepithelial PRK and aaPRK in patients who are candidates for refractive surgery showed very similar results 3 months after surgery in terms of uncorrected visual acuity, spherical equivalent and with minimal postoperative complications. TransPRK offers greater advantages to the patient regarding shorter surgical time, rapid epithelial closure and less pain in the immediate postoperative period.

Key words: PRK. Trans-PRK. Myopia. Astigmatism. Visual acuity. Spherical equivalent.

\section{Introducción}

Los procedimientos para corregir los defectos refractivos a través de la modificación de la córnea han tenido un proceso evolutivo encaminado fundamentalmente a obtener la mayor posibilidad de emetropía, predictibilidad, eficacia y seguridad. En su devenir tecnológico, las técnicas de superficie no han quedado exentas a esta evolución. Si bien el LASIK ha sido el protagonista de estos perfeccionamientos a lo largo de la historia, las técnicas de superficie también han sido objeto de investigaciones con el fin de optimizar sus resultados ${ }^{1-3}$.

El método original de remover el epitelio corneal antes de realizar la ablación con excímer láser fue durante años el mecánico (manual) y continúa utilizándose a nivel internacional con la obtención de altos estándares refractivos. En 2003, Camellin ${ }^{4}$ propone una nueva técnica asistida con alcohol absoluto llamada queratectomía subepitelial asistida con láser (LASEK), la cual preserva el epitelio, que es repuesto posterior a la ablación con láser. El láser epitelial in situ keratomileusis (Epi-LASIK) es otro método que usa el flap epitelial, en este caso con la utilización de microquerátomo ${ }^{5}$.

A finales de los años noventa, la queratectomía fotorrefractiva transepitelial (trans-PRK) comienza a surgir como una técnica en la que en un primer momento se realiza la ablación fototerapéutica del epitelio corneal para luego realizar la ablación refractiva con excímer láser del estroma corneal. En aquel momento, la técnica quirúrgica no obtuvo los resultados esperados en el camino de obtener resultados refractivos superiores, teniendo en cuenta el mayor tiempo quirúrgico del procedimiento, el mayor dolor postoperatorio del paciente y la necesidad del ajuste de nomogramas de tratamiento ${ }^{6-9}$.

El continuo desarrollo de los láseres refractivos, específicamente la disminución del tiempo de ablación y el advenimiento de los láseres rápidos, ha posibilitado un resurgir de la trans-PRK como técnica refractiva cada vez más utilizada en la actualidad, donde en un solo paso se realiza la ablación del epitelio y del estroma corneal, lo que disminuye el tiempo quirúrgico y aumenta el confort del paciente durante el procedimiento. Teniendo en cuenta estos aspectos y la adquisición de esta tecnología en nuestra institución hemos decidido realizar la actual investigación.

\section{Objetivo}

Establecer una comparación entre la técnica PRK convencional, asistida con alcohol (PRKaa), y la transPRK de un solo paso, en cuanto a variables clínico-refractivas y quirúrgicas.

\section{Método}

Se realizó un estudio observacional, prospectivo, longitudinal, en 72 pacientes candidatos a cirugía refractiva corneal con excímer láser, con el diagnóstico de astigmatismo miópico compuesto, en el Centro Oftalmológico Exiláser, Cuenca, Ecuador, de septiembre a diciembre de 2019.

\section{Criterios de selección de los casos}

Criterios de inclusión:

- Pacientes mayores de 21 años.

- Pacientes con diagnóstico de astigmatismo miópico compuesto (menor a 8 dioptrías en suma algebraica de esfera y cilindro).

- Estabilidad refractiva de 2 años.

- Agudeza visual sin corrección de 0.5 o menos.

- Agudeza visual con corrección en el ojo de menor visión superior a 0.5 .

- Lecho corneal residual programado mayor de $400 \mu \mathrm{m}$

- Queratometría media inicial y programada final entre 36 y 48 dioptrías.

- Paquimetría preoperatoria superior a $500 \mu \mathrm{m}$.

Criterios de exclusión:

- Pacientes con patologías o cirugías oculares previas (cirugía refractiva corneal, trasplante de córnea, queratitis por herpes simple, zóster, ectasia corneal 
confirmada o en sospecha, erosiones corneales recidivantes, leucomas, pannus, distrofias, degeneraciones, estrabismo o cirugía previa del mismo, glaucoma o hipertensión ocular, esclerosis del cristalino o catarata, uveítis, ojo único, desgarros, historia de desprendimiento de retina, vitrectomía, degeneración macular, retinosis pigmentaria)

- Pacientes que no asistieron a alguna de las consultas programadas en el estudio.

- Pacientes que no otorgaron su consentimiento para participar en el estudio.

- Enfermedades sistémicas como diabetes mellitus, epilepsia, enfermedades del colágeno, inmunodeprimidos, trastornos psiquiátricos, síndrome de Marfan, Ehlers Danlos, psoriasis, alergias.

- Infecciones sistémicas.

- Embarazo. Puerperio (hasta 6 meses).

- Alteración de los anexos oculares y de la lágrima (infección, inflamación, ojo seco).

- Configuraciones orbitarias anormales (órbitas pequeñas o profundas, hendidura palpebral pequeña, enoftalmos, arco superciliar prominente).

Los 72 pacientes fueron intervenidos con una técnica de superficie, de forma aleatoria sucesiva: los primeros 32 pacientes con PRKaa y después de la adquisición de la tecnología necesaria, los siguientes 40 pacientes con trans-PRK, de tal forma quedaron formados dos grupos:

- N1 = 32, pacientes operados con la técnica PRKaa.

- N2 = 40, pacientes operados con la técnica trans-PRK.

\section{Variables del estudio}

- Esfera: Se seleccionó el valor de refracción dinámica en el preoperatorio y a los 3 meses de postoperatorio.

- Cilindro: Se seleccionó el valor de refracción dinámica en el preoperatorio y a los 3 meses de postoperatorio.

- Agudeza visual sin corrección: Tomada con Cartilla de Snellen en dos momentos, preoperatorio y a los 3 meses del postoperatorio.

- Grado de haze corneal: Según la siguiente clasificación, fue evaluado por un oftalmólogo, con enmascaramiento de técnica quirúrgica empleada, en biomicroscopia anterior a los 3 meses de postoperatorio.

- Grado 0: Córnea totalmente transparente.

- Grado 1: Haze de mínima densidad, solamente visible con iluminación tangencial indirecta de la córnea.
- Grado 2: Haze ligero, que muestra áreas de confluencia focal, visible con iluminación directa de la córnea.

- Grado 3: Haze moderado, clínicamente significativo, que muestra áreas de confluencia difusa, las cuales oscurecen parcialmente los detalles del iris.

- Grado 4: Haze grave, córnea opaca que impide la apreciación del iris.

- Tiempo quirúrgico: Se midió el tiempo real de la cirugía desde la colocación del espéculo palpebral hasta su retirada, expresado en segundos.

- Tiempo de ablación: Se registró el tiempo de ablación corneal, en el caso de trans-PRK el tiempo total de epitelio y estroma, expresado en segundos.

- Dolor ocular: Se evaluó al culminar la cirugía (día 0), en consulta de postoperatorio a las 24 horas (día 1) y los días sucesivos hasta el quinto día. El paciente refirió subjetivamente, en una escala del 1 al 10 (mayor dolor), el nivel de dolor ocular al oftalmólogo en consulta.

- Tiempo de epitelización corneal: Tiempo de formación del epitelio corneal de forma íntegra posterior a la cirugía, expresado en días. Evaluado en biomicroscopio anterior el día 1, 3, 5 o 7.

- Microscopia endotelial: Obtenido en cél $/ \mathrm{mm}^{2}$ a partir del microscopio endotelial. Efectuado previo a la cirugía, al mes y a los 3 meses de postoperatorio.

\section{Examen preoperatorio}

Se obtuvo información preoperatoria sobre el historial médico general y ocular, el uso de lentes de contacto y el uso de medicamentos de cada paciente. El examen incluyó agudeza visual a distancia sin corrección (AVSC), agudeza visual a distancia con corrección (AVCC), refracción manifiesta y ciclopléjica, biomicroscopia con lámpara de hendidura, tonometría, microscopia endotelial, pupilometría, tomografía de cámara Scheimpflug (Pentacam), examen de fondo de ojo y estudio de la motilidad ocular.

\section{Técnica quirúrgica}

Todas las cirugías se realizaron con láser excímer EX500 (Suite refractiva Alcon). Los tratamientos fueron realizados por dos cirujanos, utilizando un protocolo quirúrgico idéntico. Los tiempos quirúrgicos entre cirujanos no variaron de forma significativa, con 0.12 segundos de intervariabilidad promedio. Las zonas ópticas programadas de todos los tratamientos efectuados fueron de $6.50 \mathrm{~mm}$. Los tratamientos se dirigieron todos a la emetropía (0.0). Antes de la cirugía, se instilaron 
gotas de hidrocloruro de proparacaína al $0.5 \%$ (tres veces en un intervalo de 5 minutos). En todos los casos se usó mitomicina $\mathrm{C}(0.02 \%)$ durante 20 segundos.

\section{PRKaa}

Se realizó instilación de una gota de anestésico tópico en el ojo a operar. Aislamiento del área quirúrgica con campo quirúrgico estéril y colocación del espéculo palpebral exponiendo el globo ocular. Aplicación de iodopovidona al $5 \%$ en fondos de saco conjuntivales por 3 minutos. Lavado con solución salina balanceada en fondos de saco conjuntivales. Colocación del contenedor de alcohol de $8.5 \mathrm{~mm}$ con centro en la pupila, previo marcado corneal durante 30 segundos. Lavado profuso con solución salina balanceada. Separación del epitelio corneal desde los bordes del marcador. Enfoque y aplicación del láser. Colocación de microesponja con mitomicina C $(0.02 \%)$ durante 20 segundos en el lecho estromal. Lavado profuso con solución salina balanceada. Colocación de lente de contacto blando. Instilación de una gota de moxifloxacino más dexametasona en el globo ocular.

\section{Trans-PRK}

Se realizó instilación de 1 gota de anestésico tópico en el ojo a operar. Aislamiento del área quirúrgica con campo quirúrgico estéril y colocación del espéculo palpebral exponiendo el globo ocular. Aplicación de iodopovidona al $5 \%$ en fondos de saco conjuntivales por 3 minutos. Lavado con solución salina balanceada en fondos de saco conjuntivales. Instilación de solución salina balanceada refrigerada $\left(10^{\circ} \mathrm{C}\right)$ en la córnea, secado de la misma. Enfoque en la córnea y comienzo de la ablación transepitelial programada para epitelio estándar de $55 \mu \mathrm{m}$. Pausa de 10 segundos y se continúa la ablación del estroma corneal. Lavado profuso con solución salina balanceada refrigerada $\left(10^{\circ} \mathrm{C}\right)$. Secado y colocación de microesponja con mitomicina C $(0.02 \%)$ durante 20 segundos en el lecho estromal. Lavado profuso con solución salina balanceada. Colocación del lente de contacto blando. Instilación de una gota de moxifloxacino más dexametasona en el globo ocular.

El tratamiento postoperatorio consistió en: Lágrimas artificiales (col), 1 gota cada 4 horas, tobramicina más dexametasona, 1 gota cada 4 horas hasta el mes de la cirugía. Posteriormente solo lágrimas artificiales (col) 2 veces al día hasta los 3 meses. Se realizaron controles postoperatorios a las 24 horas, 72 horas, 5 días, 7 días, al mes y a los 3 meses de la intervención. No se
Tabla 1. Equivalente esférico promedio de la muestra por técnica quirúrgica. Centro Oftalmológico Exiláser, septiembre-diciembre de 2019

\begin{tabular}{|l|r|r|c|}
\hline & PRKaa & Trans-PRK & p \\
\hline Preoperatorio & -4.17 & -3.96 & 0.16 \\
\hline Postoperatorio (1 mes) & -0.21 & -0.18 & 0.27 \\
\hline Postoperatorio (3 meses) & -0.14 & -0.11 & 0.34 \\
\hline
\end{tabular}

observaron complicaciones intraoperatorias. Se programó retirar el lente de contacto terapéutico según el tiempo de cierre epitelial.

\section{Métodos de obtención de la información, análisis estadístico y aspectos éticos}

La información fue obtenida a partir de las historias clínicas y el Pentacam de cada paciente. El procesamiento de los datos se realizó en el programa SPSS, versión 21.0. Se obtuvo la media de las variables estudiadas por cada técnica quirúrgica. Para la comparación de los datos de ambas técnicas quirúrgicas se empleó la prueba de chi cuadrado, donde se consideró $p<0.05$ estadísticamente significativo. Los pacientes otorgaron su consentimiento para la participación en el estudio y la investigación fue aprobada en el Comité de Bioética de la institución.

\section{Resultados}

Se obtuvo disminución del equivalente esférico en ambas técnicas quirúrgicas, con valores promedio cercanos a la emetropía. No se obtuvieron diferencias significativas en el promedio del equivalente esférico obtenido entre ambos procedimientos en los dos momentos postoperatorios evaluados (Tabla 1).

La tabla 2 muestra la agudeza visual sin corrección promedio de cada grupo. En ambas técnicas se obtuvieron valores cercanos al 1.0 (20/20) como media, sin diferencias estadísticamente significativas entre ambos procedimientos.

En cuanto al tiempo de ablación (Tabla 3), este fue superior en la trans-PRK con diferencia estadísticamente significativa, comparado con el tiempo de ablación promedio de la muestra en la PRKaa. Sin embargo, el tiempo del procedimiento quirúrgico fue mayor en la PRKaa, en relación con el tiempo promedio empleado en la transPRK, con diferencia estadísticamente significativa. 


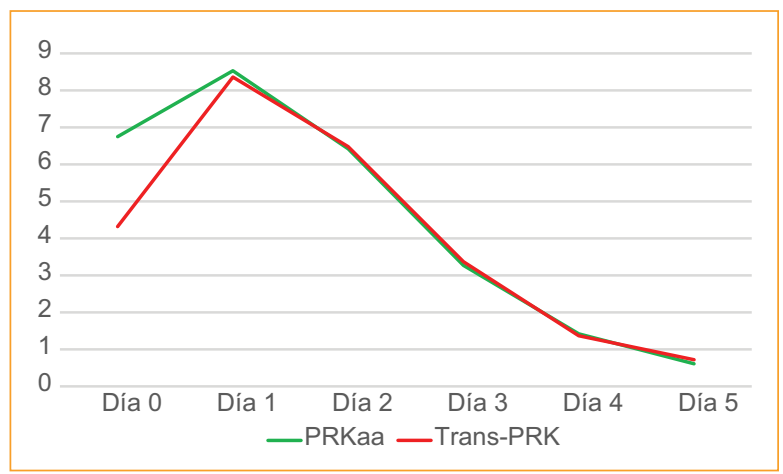

Figura 1. Curvas de dolor postoperatorio promedio por día, en ambas técnicas quirúrgicas. Centro Oftalmológico Exiláser, septiembre-diciembre de 2019.

Como muestra la figura 1, los pacientes refirieron mayor dolor en las primeras horas postoperatorias. Este fue mayor en pacientes intervenidos con PRKaa, con diferencia estadísticamente significativa, comparada con el valor promedio de dolor referido en pacientes intervenidos con trans-PRK. En ambas técnicas quirúrgicas hubo un pico máximo de dolor el primer día posquirúrgico, sin diferencias entre ambas técnicas. Hubo disminución progresiva del dolor hasta el quinto día posquirúrgico, con comportamiento muy similar en ambos procedimientos (Tabla 4).

Como se observa en la tabla 5, los pacientes intervenidos con trans-PRK tuvieron un cierre epitelial más temprano, con diferencia estadísticamente significativa comparado con los pacientes intervenidos con PRKaa.

En el postoperatorio de 1 mes no fue constatado al examen biomicroscópico la presencia de haze corneal en pacientes del estudio, sin embargo, a los 3 meses de la cirugía tres pacientes mostraban presencia de haze corneal grado I. No se reportaron otras complicaciones transoperatorias o postoperatorias durante el seguimiento de los pacientes (Tabla 6).

Como muestra la tabla 7 , no se obtuvieron variaciones significativas de la densidad celular endotelial, entre el valor promedio preoperatorio, con respecto a los valores promedios obtenidos al mes y a los 3 meses de seguimiento, en ambas técnicas quirúrgicas. No hubo diferencias significativas entre ambos grupos de pacientes intervenidos.

\section{Discusión}

Los resultados refractivos de ambas técnicas quirúrgicas fueron satisfactorios. No se obtuvieron diferencias entre ambos procedimientos, desde el mes hasta los
Tabla 2. Agudeza visual sin corrección promedio de la muestra por técnica quirúrgica. Centro Oftalmológico Exiláser, septiembre-diciembre de 2019

\begin{tabular}{|l|c|c|c|}
\hline & PRKaa & Trans-PRK & p \\
\hline Preoperatorio & 0.32 & 0.26 & 0.16 \\
\hline Postoperatorio (1 mes) & 0.87 & 0.84 & 0.55 \\
\hline Postoperatorio (3 meses) & 0.93 & 0.96 & 0.63 \\
\hline
\end{tabular}

Tabla 3. Tiempos quirúrgicos promedios por técnica quirúrgica. Centro Oftalmológico Exiláser, septiembrediciembre de 2019

\begin{tabular}{|l|c|c|c|}
\hline Tiempos (segundos) & PRKaa & Trans-PRK & p \\
\hline Ablación & 12.34 & 42.48 & $<0.001$ \\
\hline Procedimiento completo & $1,080.85$ & 720.31 & $<0.001$ \\
\hline
\end{tabular}

Tabla 4. Dolor postoperatorio promedio (máximo 10) por días posquirúrgicos. Centro Oftalmológico Exiláser, septiembre-diciembre de 2019

\begin{tabular}{|l|c|c|c|}
\hline Días & PRKaa & Trans-PRK & $p$ \\
\hline 0 & 6.75 & 4.32 & $<0.05$ \\
\hline 1 & 8.53 & 8.36 & 0.12 \\
\hline 2 & 6.42 & 6.48 & 0.23 \\
\hline 3 & 3.27 & 3.37 & 0.25 \\
\hline 4 & 1.42 & 1.37 & 0.19 \\
\hline 5 & 0.61 & 0.72 & 0.21 \\
\hline
\end{tabular}

Tabla 5. Tiempo de cierre epitelial promedio por técnica quirúrgica. Centro Oftalmológico Exiláser, septiembrediciembre de 2019

\begin{tabular}{|l|c|c|c|}
\hline & PRKaa & Trans-PRK & p \\
\hline Día promedio & 6.27 & 3.62 & 0.02 \\
\hline
\end{tabular}

Tabla 6. Pacientes con haze corneal. Centro Oftalmológico Exiláser, septiembre-diciembre de 2019

\begin{tabular}{|l|c|c|}
\hline & PRKaa & Trans-PRK \\
\hline Postoperatorio (1 mes) & 0 & 0 \\
\hline Postoperatorio (3 meses) & 1 & 2 \\
\hline
\end{tabular}


Tabla 7. Densidad celular del endotelio corneal (promedio). Centro Oftalmológico Exiláser, septiembre-diciembre de 2019

\begin{tabular}{|l|c|c|c|c|}
\hline Técnicas quirúrgicas & Preoperatorio & Postoperatorio (1 mes) & Postoperatorio (3 meses) & p \\
\hline PRKaa & 2,431 & 2,422 & 2,425 & 0.36 \\
\hline Trans-PRK & 2,426 & 2,409 & 2,432 & 0.42 \\
\hline
\end{tabular}

valores obtenidos a los 3 meses, lo que evidencia la eficacia de ambas en el tratamiento del astigmatismo miópico compuesto. Los valores de AVSC corroboran este resultado, y se alcanzaron altos estándares como promedio de la muestra en los dos grupos de estudio.

En el caso de la PRKaa, la ablación láser se realiza de forma directa y única en el estroma corneal, una vez que se ha retirado de forma mecánica el epitelio corneal, lo cual brinda una mayor seguridad en términos de exactitud del tejido estromal removido y su relación exacta con la cantidad de dioptrías a tratar, si lo comparamos con el procedimiento de la trans-PRK, teniendo en cuenta que en este procedimiento se produce una doble ablación: primero, al epitelio corneal, y después, al estroma corneal.

En la trans-PRK, el perfil de ablación se obtiene a partir de un valor de grosor epitelial de referencia, tomado como estándar para la población. El grosor del epitelio corneal de una córnea normal oscila entre 55 y $65 \mu \mathrm{m}$ en los $4 \mathrm{~mm}$ centrales. En el actual estudio tomamos como $55 \mu \mathrm{m}$ de grosor, el valor de referencia para la realización de los tratamientos. Este hecho puede resultar controversial, teniendo en cuenta que todos los pacientes no tienen el mismo grosor epitelial, por lo que en pacientes con epitelios delgados se puede ablacionar más estroma que el programado, por otra parte, en pacientes con epitelios gruesos, la ablación con fines refractivos comenzaría con presencia de epitelio en la superficie $y$, por tanto, los pacientes podrían quedar ligeramente hipo o hipercorregidos.

Diferentes autores se han pronunciado respecto a las diferencias de grosor epitelial a lo largo de la superficie corneal. Reinstein, et al. ${ }^{10}$ obtienen que la ubicación del epitelio más delgado se encuentra algo desplazado temporalmente $(0.33 \mathrm{~mm})$ y hacia la córnea superior $(0.90 \mathrm{~mm})$, muestran un grosor epitelial medio de 53.4 $\pm 4.6 \mu \mathrm{m}$ en el vértice corneal utilizando ecografía digital de muy alta frecuencia. Además, en su análisis de mapas de grosor epitelial obtuvieron mayor grosor del epitelio en la córnea inferior comparada con la córnea superior, y en la córnea nasal comparada con la temporal.
Kanellopoulos, et al. ${ }^{11}$ y Sin, et al. ${ }^{12}$ han seguido esta línea de investigación con el empleo de tomografía de coherencia óptica del segmento anterior de dominio espectral. El grosor epitelial obtenido en el centro pupilar fue de $53.28 \pm 3.34 \mu \mathrm{m}$, en la córnea inferior, de $53.81 \pm 3.44 \mu \mathrm{m}$ y en córnea superior, de $51.86 \pm$ $3.78 \mu \mathrm{m}$, por lo que concluyen que no se puede aseverar que el mapa de grosor del epitelio es rotacionalmente simétrico.

Diferentes publicaciones coinciden en la alta variabilidad interindividual del grosor epitelial central y mapas epiteliales tridimensionales ${ }^{13-15}$. Teniendo en cuenta los aspectos teóricos mencionados anteriormente, pudiera pensarse en el deterioro de los resultados refractivos en la trans-PRK si tomamos como estándar un valor de grosor epitelial único para la realización del procedimiento. Sin embargo, en la práctica, no se han logrado constatar resultados visuales insatisfactorios de esta técnica comparada con la PRKaa.

Un factor determinante en los óptimos resultados refractivos de la trans-PRK es la presencia de un perfil de ablación libre de aberración esférica. Además, el sistema láser se ajusta para compensar la diferencia entre la tasa fotoablativa del estroma y del epitelio corneal (20\% superior). El epitelio es removido con un perfil de corte de caras paralelas, es decir, se remueve en la misma proporción en toda la extensión en que se está retirando el epitelio hasta llegar a la zona de transición, donde paulatinamente deja de remover tejido. Por lo tanto, esta remoción no genera ningún efecto correctivo ${ }^{16-18}$.

Desde los inicios de la utilización de la trans-PRK en un solo paso, varios estudios mostraron los resultados preliminares. Luger, et al. ${ }^{13}$ realizaron un estudio donde en el mismo paciente se operaba un ojo con trans-PRK y el ojo contralateral con PRKaa, con el láser Amaris (Schwind), en 33 pacientes con 1 año de seguimiento. Se obtuvieron diferencias significativas entre ambas técnicas en términos de agudeza visual sin corrección. Igualmente, con la plataforma Amaris, Fadlallah, et al. ${ }^{15}$ obtuvieron parámetros de agudeza visual similares en ambas técnicas quirúrgicas. Aslanides, et al..$^{14}$ realizaron 
un estudio en 30 pacientes utilizando una técnica en cada ojo, y obtuvieron valores similares de agudeza visual en ambos procedimientos.

Kaluzny, et al. ${ }^{16}$ realizaron un estudio en 148 pacientes donde utilizan una versión más moderna de transPRK (plataforma Amaris, versión 750 S, sexta generación), comparada con los estudios citados anteriormente. No obtuvo diferencias estadísticamente significativas entre el grupo de trans-PRK y el grupo PRKaa en términos de AVSC, 3 meses después de la cirugía. Aslanides, et al..$^{14}$ no reportaron diferencias significativas en la refracción final alcanzada en ambas técnicas quirúrgicas con equivalencia en seguridad. Ortueta, et al..$^{19}$, en un estudio más actual, concluyeron que la trans-PRK tiene resultados refractivos similares a las técnicas intraestromales, con menor posibilidad de complicaciones. Xi, et al. ${ }^{20}$ demuestran que la transPRK en un solo paso puede corregir la miopía de forma efectiva.

Antonios, et al..$^{21}$ obtienen en la mayoría de la muestra de estudio pacientes que se encontraban en el rango de $\pm 0.50 \mathrm{D}$ y $\pm 1.0 \mathrm{D}$ al año del procedimiento. Fadlallah, et al. ${ }^{15}$ exponen una tendencia a la sobrecorrección que pudiera estar explicada por la deshidratación corneal durante el procedimiento. Adib-Moghaddam, et al. ${ }^{22-25}$ indican que la trans-PRK de un solo paso con modo libre de aberración mejora la agudeza visual y la refracción en los ojos miopes altos, así mismo se obtienen mejores resultados de calidad de visión comparado con otras técnicas refractivas. La plataforma de un solo paso de trans-PRK utiliza un perfil poblacional de grosor corneal para calcular la cantidad de energía entregada a diferentes partes de la córnea, lo cual evita el suministro de cantidades desiguales de energía a la córnea central en comparación con la córnea periférica. Esta diferencia podría explicar una mejor calidad de los resultados de visión de trans-PRK en la miopía alta26-29.

El tiempo de duración de la cirugía es otra de las variables ampliamente estudiadas, teniendo en cuenta su valor en la eficacia del tiempo de utilización del quirófano, la utilidad de los parámetros del láser y la comodidad del paciente durante el procedimiento. En primera instancia pudiera pensarse que la trans-PRK requiere de un mayor tiempo quirúrgico, teniendo en cuenta la ablación prolongada del epitelio corneal en comparación con la PRKaa, donde se retira de forma mecánica. Sin embargo, la utilización del contenedor de alcohol absoluto, el lavado con solución salina y la necesidad de mayor cooperación del paciente son aspectos que prolongan el tiempo quirúrgico.
Constituye una ventaja de la trans-PRK la reducción del tiempo de cirugía. Kaluzny, et al. ${ }^{16}$ obtienen una reducción (35\%) del tiempo quirúrgico en la trans-PRK comparado con PRKaa. Luger, et al. ${ }^{13}$ plantean que la trans-PRK es más rápida de realizar. Relacionado con la variable tiempo se encuentra el dolor trans y postoperatorio. La cirugía en sí es menos estresante para el paciente y muy cómoda para el cirujano. El tema del dolor postoperatorio en técnicas refractivas de superficie ha sido estudiado por diferentes autores. Fadlallah, et al. ${ }^{15}$ y Aslanides, et al..$^{14}$ informaron de una disminución del dolor postoperatorio después de la PRK transepitelial de un solo paso. Kanitkar, et al. ${ }^{7}$ obtuvieron menor dolor en la PRKaa comparado con la PRK precedida de queratectomía fototerapéutica para eliminar el epitelio corneal. Luger, et al..$^{13}$ refieren menor dolor postoperatorio en pacientes intervenidos por trans-PRK comparado con PRKaa, además reportaron mayor confort del paciente durante la cirugía. Aslanides, et al. ${ }^{14}$, sin embargo, no obtienen diferencias significativas entre ambos grupos el primer día de postoperatorio, pero, reportan menor dolor en el grupo de trans-PRK al tercer día del procedimiento.

En nuestro estudio, la mayor diferencia en cuanto a dolor referido por el paciente se produjo inmediatamente después del procedimiento, donde obtuvimos mayor valor promedio de dolor en pacientes intervenidos con PRKaa. La ausencia de alcohol en la transPRK, el menor tiempo quirúrgico, la realización de la cirugía en un solo paso y la no manipulación directa por el cirujano del globo ocular son factores que pueden explicar estas diferencias. En los días consecutivos no hubo diferencias significativas de dolor referido por el paciente, entre ambos grupos de estudio. El dolor en los días sucesivos tiene mayor relación con el efecto fotoablativo, los mediadores inflamatorios, el proceso de reepitelización corneal, comunes a ambas técnicas quirúrgicas.

En cuanto al haze corneal, en ambas técnicas se produjo, pero en una minoría de casos, todos con grado I. Fueron tratados con esteroides tópicos (fluorometalona) y sin implicaciones visuales significativas. Los defectos refractivos tratados en estos pacientes con haze no superaron las $4 \mathrm{D}$ en suma algebraica de esfera y cilindro. La energía del láser, entre otras consecuencias, provoca un aumento de la temperatura a nivel del estroma corneal, lo cual constituye uno de los principales factores relacionados con la presencia del haze, aunque se han citado otros, como la regularidad del borde del epitelio corneal receptor, la magnitud del defecto refractivo tratado, la ausencia de medicación 
tópica esteroidea postoperatoria, así como factores propios del paciente y su cicatrización ${ }^{30-33}$.

Diferentes estudios han analizado la respuesta del estroma corneal a la ablación y la consecuente formación del haze corneal. Helena, et al. ${ }^{34}$ y Kim, et al. ${ }^{35}$ plantean que la apoptosis de queratocitos y la activación de miofibroblastos son un factor clave en la recuperación del estroma después de los procedimientos de ablación de superficie, y que la ablación transepitelial produce niveles más bajos de apoptosis de queratocitos. Otros autores refieren que la eliminación del epitelio transepitelial produce una superficie uniforme y lisa, ideal para la regeneración epitelia| ${ }^{36-39}$. Los estudios de $\mathrm{Chen}^{40}$, et al. exponen el hecho de que la viabilidad de las células epiteliales del limbo se reduce con la aplicación de alcohol, con aumento de la respuesta inflamatoria y daño a los queratocitos del estroma anterior, después de la exposición al mismo.

En la trans-PRK es mayor la carga total de energía del láser excímer. Kaluzny, et al. ${ }^{16}$ refieren un tiempo medio de ablación del 163\% más largo en el grupo trans-PRK; sin embargo, se debe destacar el hecho de que la mayor parte de la energía del láser se entrega al epitelio, con previa irrigación de gotas de solución salina a $10{ }^{\circ} \mathrm{C}$ y con la pausa de 10 segundos antes de continuar a la ablación estromal, factores que atenúan el efecto de aumento de temperatura corneal en estos pacientes.

Aslanides, et al. ${ }^{14}$ plantean diferencias significativas de la presencia de haze corneal los primeros 6 meses de postoperatorio, con mayor presencia en el grupo de PRKaa. Al año de tratamiento reportan que no hubo diferencias entre ambos grupos. Kaluzny, et al. ${ }^{16}$, hasta 3 meses después de la cirugía, observaron haze con mayor frecuencia en el grupo de trans-PRK, sin diferencias estadísticamente significativas comparado con el grupo PRKaa. Las diferencias no significativas en la intensidad y presencia de haze en los dos grupos pueden explicarse por el uso de mitomicina $C$, aplicado en ambas técnicas. Sería interesante el análisis comparativo del haze en ambas técnicas sin la utilización de mitomicina $C$ para valorar el efecto real de la ablación. Esto no posible hacerlo en humanos por las evidentes implicaciones éticas.

Aslanides, et al. ${ }^{14}$ obtienen que al tercer día del postoperatorio el grupo de pacientes con trans-PRK en su mayoría mostraban cierre epitelial, con diferencias estadísticamente significativas, comparado con el grupo de PRKaa, que en su mayoría se observó cierre epitelial al quinto día del procedimiento. Fattah, et al. ${ }^{41}$ obtienen cierre epitelial más rápido con trans-PRK y menor incidencia de erosiones corneales, comparado con el grupo de PRKaa. La razón principal que explica la recuperación más rápida del epitelio corneal en trans-PRK es que el diámetro de la extirpación epitelial coincide con la zona de ablación total, lo que disminuye la superficie de la herida y acorta el tiempo de cierre epitelial21,22.

En cuanto a la densidad endotelial corneal no encontramos variaciones posquirúrgicas significativas en los dos procedimientos analizados. No encontramos evidencia científica de alteración endotelial producida por PRKaa o trans-PRK cuando se cumplen de forma óptima los protocolos establecidos para estos procedimientos.

Dentro de las limitaciones del actual estudio se encuentra la evaluación subjetiva del haze corneal en biomicroscopio anterior, siendo otros métodos más objetivos, como la microscopia confocal o la densitometría. Por otra parte, en estudios futuros sería necesario evaluar, además, las diferencias entre ambas técnicas en cuanto a aberraciones corneales y realizar medidas preoperatorias de grosor epitelial con la posibilidad de tratamientos personalizados de trans-PRK.

\section{Conclusiones}

La PRK transepitelial de un solo paso y la PRKaa realizadas en pacientes candidatos a cirugía refractiva producen resultados muy similares a los 3 meses de la cirugía en cuanto a AVSC, equivalente esférico obtenido y complicaciones postoperatorias, que son mínimas. La trans-PRK ofrece mayores ventajas al paciente, referentes a menor tiempo quirúrgico, rápido cierre epitelial y menor dolor en el postoperatorio inmediato.

\section{Conflicto de intereses}

Los autores declaran que no existe conflicto de intereses.

\section{Financiación}

Los autores declaran que no han recibido financiación para la realización de la investigación.

\section{Responsabilidades éticas}

Protección de personas y animales. Los autores declaran que los procedimientos seguidos se conformaron a las normas éticas del comité de experimentación humana responsable y de acuerdo con la Asociación Médica Mundial y la Declaración de Helsinki. 
Confidencialidad de los datos. Los autores declaran que han seguido los protocolos de su centro de trabajo sobre la publicación de datos de pacientes.

Derecho a la privacidad y consentimiento informado. Los autores han obtenido el consentimiento informado de los pacientes y/o sujetos referidos en el artículo. Este documento obra en poder del autor de correspondencia.

\section{Bibliografía}

1. Kaluzny B, Cieslinska I, Mosquera S, Verma S. Single-Step transepithelial PRK vs alcohol-assisted PRK in myopia and compound myopic astigmatism correction. Medicine (Baltimore). 2016;95(6):e1993.

2. Abad JC, An B, Power WJ, Foster CS, Azar DT, Talamo JH. A prospective evaluation of alcohol-assisted versus mechanical epithelial removal before photorefractive keratectomy. Ophthalmology. 1997;104:1566-74.

3. Dupps WJ, Jr, Kohnen T, Mamalis N, Rosen ES, Koch DD, Obstbaum SA et al. Standardized graphs and terms for refractive surgery results. Cataract Refract Surg. 2011;37:1-3.

4. Camellin M. Laser epithelial keratomileusis for myopia. J Refract Surg. 2003;19:666-70.

5. Pallikaris IG, Katsanevaki VJ, Kalyvianaki MI, Naoumidi II. Advances in subepithelial excimer refractive surgery techniques: Epi-LASIK. Curr Opin Ophthalmol. 2003;14:207-12.

6. Carr JD, Patel R, Hersh PS. Management of late corneal haze following photorefractive keratectomy. J Refract Surg. 1995;11:S309-13.

7. Kanitkar KD, Camp J, Humble H, Shen DJ, Wang MX. Pain after epithelial removal by ethanol-assisted mechanical versus transepithelial excimer laser debridement. J Refract Surg. 2000;16:519-22.

8. Clinch TE, Moshirfar M, Weis JR, Ahn CS, Hutchinson CB, Jeffrey JH Comparison of mechanical and transepithelial debridement during photorefractive keratectomy. Ophthalmology. 1999;106:483-9.

9. Lee HK, Lee KS, Kim JK, Kim HC, Seo KR, Kim EK. Epithelial healing and clinical outcomes in excimer laser photorefractive surgery following three epithelial removal techniques: mechanical, alcohol, and excimer laser. Am J Ophthalmol. 2005:139:56-63.

10. Reinstein DZ, Archer TJ, Gobbe M, Silverman RH, Coleman DJ. Epithelia thickness in the normal cornea: three-dimensional display with Artemis very high-frequency digital ultrasound. J Refract Surg. 2008;24:571-81.

11. Kanellopoulos AJ, Asimellis G. In vivo three-dimensional corneal epithelium imaging in normal eyes by anterior-segment optical coherence tomography: a clinical reference study. Cornea. 2013;32:1493-8.

12. Sin S, Simpson TL. The repeatability of corneal and corneal epithelia thickness measurements using optical coherence tomography. Optom Vis Sci. 2006;83:360-5.

13. Luger MH, Ewering T, Arba-Mosquera S. Consecutive myopia correction with transepithelial versus alcohol-assisted photorefractive keratectomy in contralateral eyes: one-year results. J Cataract Refract Surg. 2012;38:1414-23

14. Aslanides IM, Padroni S, Arba Mosquera S, loannides A, Mukherjee A. Comparison of single-step reverse transepithelial all-surface laser ablation (ASLA) to alcohol-assisted photorefractive keratectomy. Clin Ophthalmol. 2012;6:973-80.

15. Fadlallah A, Fahed D, Khalil K, Dunia I, Menassa J, El Rami H, et al. Transepithelial photorefractive keratectomy: clinical results. J Cataract Refract Surg. 2011;37:1852-7.

16. Kaluzny BJ, Szkulmowski M, Bukowska DM, Wojtkowski M. Spectral OCT with speckle contrast reduction for evaluation of the healing process after PRK and transepithelial PRK. Biomed Opt Express. 2014;5:1089-98.

17. Adib-Moghaddam S, Arba-Mosquera S, Salmanian B, Omidvari AH, Noorizadeh $\mathrm{F}$. On-line pachymetry outcome of ablation in aberration free mode TransPRK. Eur J Ophthalmol. 2014;24:483-9.

18. Arba Mosquera S, Awwad ST. Theoretical analyses of the refractive implications of transepithelial PRK ablations. $\mathrm{Br} \mathrm{J}$ Ophthalmol. 2013;97:905-11.

19. Ortueta D, von Rüden D. Transepithelial photorefractive keratectomy: Results and clinical experiences. Ophthalmologe. 2019;116(6):534-41.
20. Xi L, Zhang C, He Y. Single-step Transepithelial photorefractive keratectomy in the treatment of mild, moderate, and high myopia: six-month results. BMC Ophthalmol. 2018;18(1):209.

21. Antonios R, Abdul Fattah M, Arba Mosquera S, Abiad BH, Sleiman K, Awwad ST. Single-step transepithelial versus alcohol-assisted photorefractive keratectomy in the treatment of high myopia: a comparative evaluation over 12 months. Br J Ophthalmol. 2017;101(8):1106-12.

22. Adib-Moghaddam S, Haydar AA, Razi-Khosroshahi M, Soleyman-Jahi S, Tefagh G, Grentzelos MA, et al. Predictors of visual acuity improvement and supernormal vision after refined single-step transepithelial photorefractive keratectomy. J Refract Surg. 2019;35(12):771-80.

23. Adib-Moghaddam S, Soleyman-Jahi S, Adili-Aghdam F, Arba Mosquera S, Hoorshad N, Tofighi S. Single-step transepithelial photorefractive keratectomy in high myopia: qualitative and quantitative visual functions. Int J Ophthalmol. 2017;10(3):445-52.

24. Adib-Moghaddam S, Soleyman-Jahi S, Sanjari Moghaddam A, Hoorshad N, Tefagh G, Haydar AA, et al. Efficacy and safety of transepithelial photorefractive keratectomy. J Cataract Refract Surg. 2018;44(10):1267-1279.

25. Adib-Moghaddam S, Arba-Mosquera S, Walter-Fincke R, Soleyman-Jahi S, Adili-Aghdam F. Transepithelial photorefractive keratectomy for hyperopia: a 12-month bicentral study. J Refract Surg. 2016;32(3):172-80.

26. Shetty N, Dadachanji Z, Narasimhan R, Kundu G, Khamar P, Ahuja P, et al. Status of residual refractive error, ocular aberrations, and accommodation after myopic LASIK, SMILE, and TransPRK. J Refract Surg. 2019;35(10):624-31.

27. Luger MH, Ewering T, Arba-Mosquera S. Myopia correction with transepithelial photorefractive keratectomy versus femtosecond assisted laser in situ keratomileusis: one-year case-matched analysis. J Cataract Refract Surg. 2016;42(11):1579-87

28. Aslanides IM, Georgoudis PN, Selimis VD, Mukherjee AN. Single-step transepithelial ASLA (SCHWIND) with mitomycin-C for the correction of high myopia: long term follow-up. Clin Ophthalmol. 2014;9:33-41.

29. Aslanides IM, Kymionis GD. Trans advanced surface laser ablation (TransPRK) outcomes using Smart Pulse Technology. Cont Lens Anterior Eye. 2017;40(1):42-6.

30. Kumar NR, Khamar P, Shetty R, Sharma A, Shetty N, Pahuja N, et al. Identification of novel predictive factors for post-surgical corneal haze. Sci Rep. 2019;9(1):16980.

31. Arranz-Marquez E, Katsanos A, Kozobolis VP, Konstas AGP, Teus MA. A critical overview of the biological effects of mitomycin $C$ application on the cornea following refractive surgery. Adv Ther. 2019;36:786-97.

32. Kaiserman I, Sadi N, Mimouni M, Sela T, Munzer G, Levartovsky S. Corneal breakthrough haze after photorefractive keratectomy with mitomycin C: Incidence and risk factors. Córnea. 2017:36:961-66.

33. Ang BC, Foo RC, Lim EW, Tan MM, Nah GK, Thean LS, et al. Risk factors for early-onset corneal haze after photorefractive keratectomy in an Asian population: Outcomes from the Singapore Armed Forces Corneal Refractive Surgery Programme 2006 to 2013. J Cataract Refract Surg. 2016;42(5):710-6.

34. Helena MC, Baerveldt F, Kim WJ, Wilson SE. Keratocyte apoptosis after corneal surgery. Invest Ophthalmol Vis Sci. 1998;39(2):276-83.

35. Kim BZ, Jordan CA, McGhee CN, Patel DV. Natural history of corneal haze after corneal collagen crosslinking in keratoconus using Scheimpflug analysis. J Cataract Refract Surg. 2016:42(7):1053-9.

36. Reinstein DZ, Archer TJ, Gobbe M. Change in epithelial thickness profile 24 hours and longitudinally for 1 year after myopic LASIK: three-dimensional display with Artemis very high-frequency digital ultrasound. J Refract Surg 2012;28:195-201.

37. Kanellopoulos AJ, Asimellis G. Longitudinal postoperative Lasik epithelial thickness profile changes in correlation with degree of myopia correction. J Refract Surg. 2014;30:166-71.

38. Raviv T, Majmudar PA, Dennis RF, Epstein RJ. Mytomycin-C for postPRK corneal haze. J Cataract Refract Surg. 2000;26:1105-6.

39. Fantes FE, Hanna KD, Waring GO, III, Pouliquen Y, Thompson KP, Savoldelli M. Wound healing after excimer laser keratomileusis (photorefractive keratectomy) in monkeys. Arch Ophthalmol. 1990;108:665-75.

40. Chen X, Stojanovic A, Hua Y, Eidet JR, Hu D, Wang J, et al. Reliability of corneal dynamic Scheimpflug analyser measurements in Virgin and post-PRK eyes. PLoS ONE. 2014;9(10):e109577.

41. Fattah MA, Antonios R, Arba Mosquera S, Abiad B, Awwad ST. Epithelial erosions and refractive results after single-step transepithelial photorefractive keratectomy and alcohol-assisted photorefractive keratectomy in myopic eyes: a comparative evaluation over 12 Months. Cornea. 2018;37(1):45-52 